\title{
REVERSE HILBERT TYPE INEQUALITIES
}

\section{CHANG-JiAN ZHAO AND WING-SUM CHEUNG}

Abstract. In this paper, some reverse Hilbert-Pachpatte's type inequalities involving series of nonnegative terms are established, which provide new estimates on inequality of this type.

Mathematics subject classification (2010): 26D15.

Keywords and phrases: Convex function, concave function, Jensen's inequality, Hilbert's inequality.

\section{REFERENCES}

[1] G. H. Hardy, J. E. Littlewood and G. Pólya, Inequalities, Cambridge Univ. Press, Cambridge, 1934.

[2] D. S. Mitrinović, Analytic Inequalities, Springer-Verlag, Berlin, New York, 1970.

[3] B. C. YAng, On Hilbert's integral inequality, J. Math. Anal. Appl., 220 (1988), 778-785.

[4] M. Z. GAO, B. C. YAng, On the extended Hilbert's inequality, Proc. Amer. Math. Soc., 126 (1998), $751-759$.

[5] J. C. Kuang, L. Debnath, On Hilbert type inequalities with non-conjugate parameters, Appl. Math. Lett., 22 (2009), 813-818.

[6] M. KRniĆ, J. PEČARIĆ, Extension of Hilbert's inequality, J. Math. Anal. Appl., 324 (2006), 150-160.

[7] Z. X. Lv, M. Z. GaO, L. Debnath, On new generalizations of the Hilbert integral inequality, J. Math. Anal. Appl., 326 (2007), 1452-1457.

[8] G. A. Anastassiou, Hilbert-Pachpatte type fractional integral inequalities, Math. Compu. Mode., 49 (2009), 1539-1550.

[9] J. Jin, L. Debnath, On a Hilbert-type linear series operator and its applications, J. Math. Anal. Appl., 371 (2010), 691-704.

[10] B. C. YANG, A half-discrete Hilbert-type inequality with a non-homogeneous kernel and two variables, Mediterranean J. Math., 10(2) (2013), 677-692.

[11] G. D. Handley, J. J. Koliha And J. E. PeČARIĆ, New Hilbert-Pachpatte type integral inequalities, J. Math. Anal. Appl., 257 (2001), 238-250.

[12] Z. T. XIE, A new reverse Hilbert-type inequality with a best constant factor, J. Math. Anal. Appl., 343 (2008), 1154-1160.

[13] C. J. Zhao, L. Debnath, Some new inverse type Hilbert integral inequalities, J. Math. Anal. Appl., 262 (2001), 411-418.

[14] T. BAtBOLD, L. E. AZAR, M. KRnić, A unified treatment of Hilbert-Pachpatte-type inequalities for a class of non-homogeneous kernels, Appl. Math. Comput., 343 (2019), 167-182.

[15] B. G. PAChPATte, On some new inequalities similar to Hilbert's inequality, J. Math. Anal. Appl., 226 (1998), 166-179.

[16] M. Tominaga, Specht's ratio in the Young inequality, Sci. Math. Japon., 55 (2002), 538-588. 